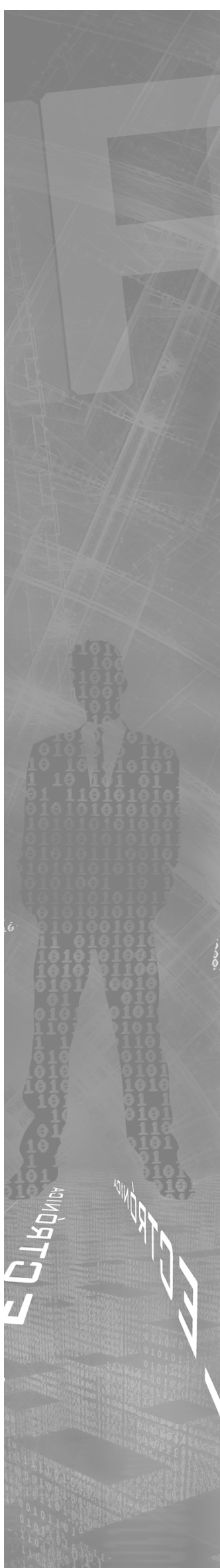

\title{
Uso de la cascarilla de arroz como material alternativo en la construcción
}

Andrés Mafla $B$.

Recibido el 19 de febrero de 2009. Aprobado el 4 de mayo de 2009

\section{Resumen}

El cemento Portland es un material que se utiliza ampliamente en la construcción de viviendas, puentes, vías, entre otras aplicaciones. En la actualidad existe un notable interés en la búsqueda de materiales cementantes que permitan mejorar la resistencia mecánica, la respuesta al ataque de los ácidos, y que favorezcan ciertas propiedades funcionales del concreto como la conductividad eléctrica, el apantallamiento contra la radiación electromagnética, y la radiación ionizada, entre otras. De otra parte, con el fin de reducir costos en la producción del cemento, reutilizar desechos industriales y la búsqueda de cementos con características especiales, se viene proponiendo la adición de algunos de estos mismos deshechos para aprovechar la naturaleza puzolánica que algunos presentan. En este documento se estudia la adición del SiO2, obtenido de la cascarilla de arroz, al Cemento Portland común y el efecto que esta materia prima tiene sobre el comportamiento mecánico del concreto fabricado con ella. Se indican las características más importantes del SiO2 que se obtuvo en el laboratorio de la Universidad del Cauca y las curvas de resistencia a la compresión de muestras de mortero.

\section{Palabras clave}

Cemento, Concreto, Clinker, Silicatos, Oxidos, Puzolanas,

\begin{abstract}
Portland cement is an extensively used material in housing, bridges and roads construction, among other applications. Nowadays, there is a notable interest in searching for cementing materials that improved mechanical resistance, reaction to acids, and that fovored certain functional properties of concrete, such as electric conductivity and protection against electromagnetic radiation and ionized radiation. In other hand, with the purpose of reduce costs in cement production, reuse industrial wastes and searching for cements with special characteristics, it has been proposed to add some of these wastes in order to make good use of the puzolanic nature some of them present. In this document, we study the addition of SiO2, obtained from rice dried skin, to Portland Cement and the effect of this prime matter on the mechanical behavior of the concrete made with it. We indicate the most important characteristics of SiO2 we obtained in the laboratory of Universidad del Cauca and resistance to compression curves of mortar samples.
\end{abstract}

\section{Key words}

Cement, Concrete, Clinker, Silicates, Oxides, Puzolanes, 


\section{Introducción}

El cemento es un material que se utiliza ampliamente en la fabricación de hormigones y morteros en obras civiles (Taylor, 1978). El cemento cuando se mezcla con el agua y los materiales granulares, tales como la arena, grava, etc. Forma un compuesto que comienza un proceso físico-químico que se denomina Fraguado. Este consiste en el endurecimiento del material a causa de las reacciones químicas que se producen entre las fases presentes. Actualmente, se estudian otras posibilidades de uso para el cemento: Fabricación de estructuras protectoras contra radiación electromagnética (Fu \& Chung, 1996), concretos reflectores de ondas de radio (Fu \& Chung, 1996), aplicaciones donde la conductividad eléctrica del concreto es importante (Tumidasjki, 1996), cementos y concretos para estructuras que apantallen contra la radiación ionizada (Rajczyk \& Wezelink, 1994), entre otras muchas aplicaciones (Odler F., 2000).

Dentro de los cementos hidráulicos se destaca el cemento Portland. Este se obtiene de la molienda del clinker, producto que resulta del tratamiento térmico a temperaturas cercanas al fundido parcial de las materias primas (arcillas y calizas) que contienen $\mathrm{CaO}, \mathrm{SiO}_{2}, \mathrm{Al}_{2} \mathrm{O}_{5}$ y Fe $\mathrm{O}_{3}$ (más otros óxidos en pequeñas cantidad) (Taylor, 1978 \& Odler, 2000). Dos de las principales fases que conforma el clinker son: El silicato tricalcico $\left(3 \mathrm{CaO} . \mathrm{SiO}_{2} \mathrm{O} \mathrm{Ca}_{3} \mathrm{SiO}_{2}\right.$ que abrevia $\left.\mathrm{C}_{3} \mathrm{~S}\right)$ y el silicato dicálcico $\left(2 \mathrm{CaO} \mathrm{SiO}_{2} \mathrm{O}_{\mathrm{CaSiO}_{4}}\right.$ que se abrevia $\mathrm{C}_{2} \mathrm{~S}$ ). El $\mathrm{C}_{3} \mathrm{~S}$ es estable termodinámicamente entre $1250^{\circ} \mathrm{C}$ y $2150^{\circ} \mathrm{C}$ y se funde incongruentemente por encima de esta última temperatura. Por debajo de la temperatura de $1250^{\circ} \mathrm{C}$ el $\mathrm{C}_{3} \mathrm{~S}$ es inestable termodinámicamente con relación al $\mathrm{C}_{2} \mathrm{~S}$ y al CaO. Por lo tanto para producir clinker que contenga silicato tricalcico, la materia prima se debe calentar a una temperatura mayor de $1250^{\circ} \mathrm{C}$ que es el limite inferior de estabilidad de esta fase y enfriarla rápidamente para impedir la descomposición del $\mathrm{C}_{3} \mathrm{~S}$. El clinker se dopa con otros iones tales como $\mathrm{Al}^{3+} \mathrm{y} \mathrm{Mg}_{2}+$ con el fin de evitar la formación de la fase triclínica del $\mathrm{C}_{3} \mathrm{~S}_{\text {, }}$ fase meta estable a temperatura ambiente, y favorecer la presencia de una de las fases monoclínicas de alta temperatura, la denominada alita (Odler F., 2000). En contacto con el agua el $C_{3} S$ se hidrata produciendo un silicato de calcio amorfo hidratado, fase C-S-H cuya fórmula general es el $\mathrm{CaO}_{x} \ldots$ $\mathrm{SiO}_{2} \mathrm{H}_{2} \mathrm{O}_{9}$, e hidróxido de calcio. Este último está presente en las pastas de cemento portland en forma de cristales entre 10-30 $\mu \mathrm{m}$ con una forma cristalina denominada portlandita.

El otro componente importante del Cemento Portland es el ortosilicato de calcio, $\mathrm{C}_{2} \mathrm{~S}$. Este puede pre- sentar cinco formas polimorfas y todas se producen en las reacciones en estado sólido entre el $\mathrm{CaO}$ y el $\mathrm{SiO}_{2}$ (Taylor 1978 \&, Odler 2000). En una reacción de compuestos puros la forma monoclínica del $\mathrm{C}_{2} \mathrm{~S}$ es la única que se produce y para obtener otras fases es necesario adicionar ciertos óxidos como dopantes (Taylor, 1978 \& Odler, 2000).

La fase monoclínica del $\mathrm{C}_{2} \mathrm{~S}$ es la fase estable a temperatura ambiente, la reacción de hidratación de esta fase es muy pobre y la velocidad de reacción es muy lenta. Por otro lado, el polimorfo mas reactivo, y por lo tanto el más importante en la química de los cementos, es el $\beta-C_{2}$ S el cual es muy inestable a cualquier temperatura. A temperaturas ambiente se transforma en la fase monoclínica.

Estudios amplios se están realizando para determinar que secuencias se pueden incorporar, (en mínima cantidad) en la estructura del silicato para estabilizar la fase $\beta$ (Kantro \& Weise 1979). Entre los óxidos empleados se destacan el $\mathrm{MgO}, \mathrm{Al}_{2} \mathrm{O}_{3}, \mathrm{P}_{2} \mathrm{O}_{5}$ y el $\mathrm{B}_{2} \mathrm{O}_{3}$ (Pritts, 1976; Taylor, 1978 \& Odler, 2000). También se ha trabajado en la posibilidad de que el tamaño de cristal del $\mathrm{C}_{2} \mathrm{~S}$ permita estabilizar la fase $\beta$ (Taylor, 1978). Los productos de hidratación del silicato dicálcico son idénticos, o casi idénticos, los que se forman durante la hidratación del $\mathrm{C}_{2} \mathrm{~S}$ : realmente la formación de hidróxido de calcio es menor (Odler, 2000). La velocidad de hidratación del $\mathrm{C}_{2} \mathrm{~S}$ es significativamente menor que la $\mathrm{C}_{3} \mathrm{~S}$ y la reacción de velocidades está determinada por el dopante que se utilice y la velocidad de enfriamiento.

De otra parte y con el fin de abaratar costos en la producción de los cementos se han venido utilizando deshechos industriales y agrícolas que presentan propiedades puzolanicas (Taylor, 1978 \&, Odler, 2000). Los materiales puzolanicos son materiales siliceos y/o aluminosos que no poseen alto poder cementante en sí mismo pero, si ellos presentan un tamaño de partícula muy pequeño y por lo tanto alta reactividad, pueden reaccionar químicamente con el $\mathrm{Ca}(\mathrm{OH})_{2}$ a temperaturas ambiente y formar compuestos que presenten propiedades cementantes. Uno de los materiales puzolanicos que se presenta es la ceniza de arroz (Páez \& Ahumada, 2006). $\mathrm{El} \mathrm{SiO}_{2}$ amorfo altamente reactivo, presente en la ceniza, reacciona con el $\mathrm{Ca}(\mathrm{OH})_{2}$ que se produce durante la hidratación del $\mathrm{C}_{3} \mathrm{~S}$ y el $\beta-\mathrm{C}_{2} \mathrm{~S}$. Al adicionar ceniza de la cascarilla de arroz se favorece la formación de más fase C-S-H (Páez \& Ahumada, 2006).

Los resultados obtenidos al adicionar $\mathrm{SiO}_{2}$, obtenidos de la cascarilla de arroz al Cemento Portland normal Tipo I, indican el efecto de esta incorporación sobre 
resistencia a la compresión del concreto fabricado con esta mezcla. Se muestra además detalles del proceso de obtención del $\mathrm{SiO}_{2}$ y características físico química y físico mecánicas del producto.

\section{Materias primas y experimentos}

\section{Obtención del SiO2}

$\mathrm{El} \mathrm{SiO}_{2}$ que se utilizó en el proyecto del cual el presente documento es resumen se obtuvo de la cascarilla de arroz a través del proceso que se denominó "proceso de reflujo".

El proceso de reflujo consiste en una serie de pasos y procesos químicos y físicos, con el fin de separar la parte orgánica de la parte inorgánica de la cáscara de arroz, ya que el cemento como compuesto puramente inorgánico tendría fenómenos de inestabilidad frente a compuestos orgánicos, causando efectos adversos a este, como perdida de la resistencia mecánica, ataque de sulfatos, asentamientos o dificultad en la manipulación del concreto fresco instancias que afectan las propiedades finales del concreto. El proceso se inicia con el lavado de la cascarilla de arroz, retirando de ella toda clase de solido para posteriormente colocarla a secar al medio ambiente. Con la cascarilla seca, se procede al tratamiento químico sometiéndola al proceso de reflujo químico en una disolución de ácido clorhídrico $\mathrm{HCl}$ por aproximadamente 5 horas. Al término de este proceso, la cascarilla de arroz se deja secar nuevamente al medio ambiente. Posteriormente se coloca en un crisol y se trata térmicamente a $700^{\circ} \mathrm{C}$ por aproximadamente 3 horas a una velocidad de calentamiento de $250^{\circ} \mathrm{C} /$ hora. Al finalizar el tratamiento, el producto sólido de color blanco, se mueve utilizando un molido de bolas, hasta obtener una superficie especifica determinada y se procede a mezclar con el mortero o concreto como tal. El proceso de molienda es muy importante, ya que una molienda excesiva puede hacer que el producto final tenga altas energías superficiales que hagan que tomen otros compuestos altamente reactivos de la pasta de cemento que afecten las propiedades finales del mortero o concreto.

Finalmente el proceso de reflujo requiere una inversión menor haciendo de esta una oportunidad en la búsqueda de nuevas alternativas de construcción y desarrollo tecnológico para países pobres y con poca industrialización.

\section{Ensayos mecánicos}

La resistencia a la compresión de los compositos obtenidos, mezcla de dióxido de silicio y mortero se determinó de acuerdo con la norma ASTM C 109-80 y la Norma Técnica Colombiana NTC 220, que es la que emplean todas las empresas de consultoría e interventoría para hacer el respectivo control de calidad de este tipo de materiales.

Para la realización de los ensayos de resistencia a la compresión, inicialmente se mezcló el cemento con el producto del reflujo de la cascarilla de arroz y una cantidad de agua adecuada de tal manera que la relación entre agua y el cemento estuviese en el valor recomendado por la Norma Colombiana Sismoresistente NSR-98 Capítulo 3 que es de 0.485. Esto se hace para rectificar con la ley de Abramhs que establece que esta relación afecta de manera directa la resistencia mecánica de los concretos y los morteros. Para comenzar con el estudio se procedió a formar cubos de cinco (5) centímetros de lado que fueron empleados en la realización del ensayo de compresión utilizando un equipo mecánico o hidráulico, aplicándole una carga que se mide con una exactitud de $+/-1.0 \%$.

Es importante resaltar que hoy por hoy las construcciones mundiales están requiriendo aún más exigencias del tipo de durabilidad. Esto ha hecho que estos materiales sean más exigentes en cuanto a sus ataques químicos, por lo tanto se debe considerar que este es otro ensayo importante a realizar, ya que la investigación estuvo enfocada inicialmente a la búsqueda de mejoramiento de la resistencia mecánica y no se consideró el buen efecto que puede tener este sobre la resistencia a la durabilidad. En este sentido, resulta importante que se continué con la medición de este fenómeno, ya que si bien hoy en día las estructuras no presentan fallas estructurales que no se puedan solventar, sí se han detectado fallas de tipo químico, las cuales afectan directamente en la pasta de los concretos o morteros y conducen a que estas deban ser demolidas parcial o totalmente, convirtiendo este fenómeno en elemento de enorme complejidad e importancia para ser considerado como prioritario en todo Colombia, como lo es ya en otras partes del mundo.

\section{Resultados y discusión}

El dióxido de silicio, que se obtiene al final del proceso se encuentra en un estado amorfo y altamente cristalino, esto fue demostrado por análisis de difracción de rayos $X$ realizados en los laboratorios de espectroscopia de las Universidad del Valle y la Universidad Nacional, Sede de Manizales. Estas partículas tienen un tamaño entre un micrón $(1 \mu)$ y cinco $(5 \mu)$ micrómetros, con una alta distribución de tamaños y con una morfología irregular, la concentración de 
impurezas es muy baja, destacándose entre estas impurezas el contenido del hierro, el cual se deba a la interacción entre el material y los elementos de la molienda, el valor de la superficie especifica oscila alrededor de los $260 \mathrm{~m} 2 / \mathrm{g}$.

Este valor indica un alto poder reactivo del material, el cual puede aportar de manera directa a la propiedad mecánica de resistencia.

Las características anteriormente indicadas hacen que el dióxido de silicio, $\mathrm{SiO}_{2}$, obtenido de la cascarilla de arroz se deba considerar como buen candidato a utilizar como aditivo del Cemento Portland tradicional. La alta reactividad del $\mathrm{SiO}_{2}$, justificada por el alto valor de su superficie especifica, favorece su reacción con el calcio libre o $\mathrm{Ca}(\mathrm{OH})_{2}$, existente en el cemento portland a temperatura ambiente y en presencia de agua. Esta reacción debe favorecer la conformación de la fase amorfa C-S-H, tal como otros autores lo han referenciado (Páez \& Ahumada, 2006).

Los resultados de la resistencia a la compresión de las muestras en forma de cubo, conformados y realizados conforme a las normas existentes y colocados durante un día en una cámara húmeda indican un mejoramiento en cuanto a su resistencia a compresión. Los porcentajes de adición oscilaron entre un 5-10\% del contenido total de cemento, reemplazando esta cantidad para revisar el verdadero comportamiento mecánico de este material.

De acuerdo con los resultados obtenidos para las muestras analizadas, se encuentra que la resistencia se incrementa notablemente, más para la primera que para la segunda, con relación a las muestras que contienen al cemento $\sin \mathrm{SiO}_{2}$. Esto se puede entender considerando que la cantidad de hidróxido de calcio, o calcio libre existente en el cemento portland disminuye debido a su reacción puzolanica con el dióxido de silicio adicionado, favoreciendo la densificación de la zona interfacial. Estudios de resistencia a la compresión para más días de envejecimiento y otras composiciones demostraron que los resultados son coherentes y la estabilidad de este compuesto es excelente.

Se encontró que la relación de agua con cemento (a/c), utilizada en la elaboración de concretos que contienen dióxido de silicio es muy importante, si esto no es así, se producen grandes diferencias de densificación en la pasta envejecida, generándose aglomerados muy duros, de tamaño aproximado de cinco milímetros ( $5 \mathrm{~mm}$ ) en el interior de la muestra y ocasionando una disminución abrupta de la resistencia a la compresión.

\section{La cascarilla de arroz y los nuevos materiales}

La cascarilla de arroz es un deshecho agroindustrial que se produce en altos volúmenes en los lugares donde se siembra y se procesa la planta del arroz. Este deshecho, como se demostró se puede utilizar para la obtención de dióxido de silicio para el cemento y mejorar sus características mecánicas lo cual ha sido investigado por especialistas en distintas partes del mundo, los cuales han notado el enorme potencial de este material como fuente alternativa en el campo de la construcción (Páez \& Ahumada 2006), esto permite establecer que para el caso concreto de Colombia también se le considere como un material de gran potencial ya que ofrece una alternativa de alta viabilidad para las construcciones debajo costo.

Con base en lo anterior, entidades como la Universidad del Valle, específicamente el departamento de nuevos materiales, han desarrollado técnicas para industrialización del proceso de la cascarilla de arroz con el fin de colocar este material como una alternativa para mejorar la resistencia mecánica y contra los sulfatos que puede sufrir un concreto puesto en obra.

La composición química de la cascarilla de arroz se la puede establecer según la tabla Nol.

\begin{tabular}{|c|c|c||c|}
\hline COMPUESTO & $\mathrm{SiO}_{2}(\%)$ & $\mathrm{CO}_{2}(\%)$ & $\mathrm{AlO}_{3}(\%)$ \\
\hline \hline Superficie Externa & 55.25 & 44.77 & 0.00 \\
\hline \hline Superficie Interna & 35.48 & 58.24 & 6.27 \\
\hline
\end{tabular}

Tabla 1 Composición Química de la Cascarilla de Arroz. Fuente: Paez 2006

Es importante resaltar que la aplicación de los silicatos se encuentran en la industria cerámica, fabricación de cerámicas tenaces y bio-cerámicas, en fundiciones metalúrgicas como aditivo fundente o simplemente como carga o recubrimiento en la fabricación de porcelanas dieléctricas, en las pinturas, en la conformación de vidrios bioactivos, en la producción de resinas y plásticos, como material reforzante mejorando las propiedades físicas, químicas, eléctricas entre otras del producto acabado.

Una alternativa a corto plazo que se visualiza, es el uso de este material como compuesto de cubiertas de poco peso. Otra aplicación que se puede abordar con el uso de este material, esta en el hecho de utilizarlo en la industria ladrillera, lo cual por medio de sinterización de los elementos constituyentes puede generar ladrillos más económicos y más livianos. De esta forma, se disminuyen los pesos de las estructuras, se aligeran las presiones sobre los suelos de fun- 
dación y finalmente se evitan los daños colaterales que se presentan durante los sismos, sobre el argumento que durante un sismo un porcentaje amplio de muerte se debe principalmente a la caída de elementos tipos muros divisorios sobre los residentes de una edificación.

\section{Conclusiones}

- El dióxido de silicio obtenido de la cascarilla de arroz, desarrollado en la Universidad del Cauca presentó buenas propiedades como material puzolanico. Su alta pureza y su alta superficie especifica hacen que al mezclarlo con el cemento portland y en presencia de agua reaccione fácilmente con el calcio o $\mathrm{Ca}(\mathrm{OH}) 2$ libre.

La disminución de dióxido de calcio $\mathrm{Ca}(\mathrm{OH}) 2$ en la zona interfacial se puede deber a la reacción de éste con el dióxido de silicio o $\mathrm{SiO} 2$ adicionado, ocasionando un incremento de la densificación del concreto en esta zona y por lo tanto un aumento de la resistencia a la compresión del mismo.

Una inadecuada relación agua - cemento ocasiona la formación de agregados esféricos muy duros en el interior del concreto y por lo tanto una abrupta disminución de la resistencia a la compresión.

El uso industrial que se le puede proporcionar a corto plazo radica en el hecho de generar nuevas alternativas de construcción haciendo vivienda de menor costo con funcionalidad adecuada.

Uno de los campos de uso es la vivienda de interés social, la cual requiere bajos costos de productos y operación

La misma condición de amorficidad de la estructura sugiere que este material se pueda utilizar tanto en la Industria del Concreto como en la Ladrillera, ya que se garantiza alta reactividad del material con los silicatos, además que puede servir como catalizador o activador en los efectos de sinterización (Cocción del ladrillo) que pueden mejorar las condiciones de medio ambiente alrededor de estas industrias.

- El uso de la cascarilla de arroz sin un previo tratamiento químico es un agente patógeno para el concreto, ya que este contiene un gran porcentaje de materia orgánica que impide el correcto fraguado de la pasta de concreto, por lo tanto siempre se debe considerar eliminar la parte orgánica no solamente por el proceso de quemado sino por el proceso de reflujo.

\section{Referencias}

[1] Arcos, C. A, Pinto, D. M. \& Paéz, J. E. (2007). La cascarilla de arroz como fuente de $\mathrm{SiO}_{2}$. Revista Facul- tad de Ingeniería, Universidad de Antioquia, No 41, pp 7-20. Medellín.

[2] Bizzoto,M.B. \& Natalini, M.B., (1998). Minihormigones con cascarilla de arroz natural y tratado con agregado natural. Primer congreso natural de tecnología, Buenos Aires, Argentina

[3] Fu. X \& Chung D.D.L., (1996). Radio - wave- reflecting concrete for lateral guidance in automatics highways. Cement and Concrete Research, Vol 28, 539 - 544

[4]Fu. X, Chung \& D.D.L., (1996). Submicron carbón filament cement - matrix, composites for electromagnetic interference shielding. Cement and Concrete research, Vol 26, 1467 - 1472

[5] González de la Cotera, M. (s.f.). Morteros ligeros de cáscara de arroz. Facultad de Ingenieria, Universidad nacional del Perú.

[6] Kantro, D. L. \& Weise, C. H., (1979). Hydratation of varius beta-dicalcium preprations, Journal American Ceramic Society, Vol 62 (11-12), 621 - 626

[7] Mafla, A., Andrade, G. \& Paez, J. E., (2002). Producción y caracterización de películas cerámicas de $\mathrm{SiO}_{2}-\mathrm{TiO}_{2}$ para su protección contra la corrosión, SLAFES, XV, Mérida, Venezuela

[8] Mafla, A., Andrade, G. \& Paéz, J. E., (2002). Obtención de soles estables de sílice - Titania, utilizando como acomplejante acetilacetonato. Revista Lati. Metal, Vol 22, N1, pp $16-20$

[9] Mafla, A., Paéz, J. E. \& Andrade, G., (2004). Modificación química del precursor de titanio para obtener soles estables de sílice titania: Uso de acetilcetona, Bol. soc. Esp. Ceram V, Vol 43 N 1, pp 53-55

[10] Odler, (2000). "special inorganics cements", Modern concrete technology, E\&FN spon, Taylor \& Francis Group.

[1 1 ] Paez, J.N \& Ahumada, L., (2006). Uso del $\mathrm{SiO}_{2}$ obtenido de la cascarilla de arroz en la síntesis de silicatos de calcio. Revista Academia Colombiana de Ciencias, 30 (117) 581 - 594. Bogotá

[12] Pritts, J.M. \& Daugherty K.E., (1976). The effect of stabilizing agents on the hydratation rate of $\mathrm{C}_{2} \mathrm{~S}, \mathrm{Ce}$ ment and Concrete Research Vol 6, $783-796$

[13] Rajczyk K., Mocun \& Wezelink W., (1994). Studies of belite cement from barium containing by-product, in proceeding 9th ICCC, Vol 2 pp $250-254$

[14] Taylor, H.F., (1978). La química de los cementos Vol 2. Enciclopedia de la química industrial. Urmo S.A. ediciones,

[15] Taylor, H.F., (1978). La química de los cementos. Vol 1. Enciclopedia de la química industrial.Urmo S.A de ediciones, 1978

[16] Tumidasjki, P.J. et al, (1996). On the relationships between porosity and electrical resistivity in cementitionus system, Cement and Concrete Research Vol 26, 539 -544

Andrés Mafla B. Ingeniero Civil, Docente Programa de Ingeniería Civil, Corporación Universitaria Minuto de Dios (UNIMINUTO) 